\title{
Breast-feeding patterns of ethnic groups in rural western China
}

\author{
Pengfei Qu ${ }^{1}$, Ting Wang ${ }^{1}$, Fang Liu ${ }^{1}$, Shaonong Dang ${ }^{1}$, Lengxia Zeng ${ }^{1}$ and Hong Yan ${ }^{1,2, *}$ \\ 'Xi'an JiaoTong University, Health Science Center, Xi'an, Shaanxi Province 710061, People's Republic of China: \\ ${ }^{2}$ Nutrition and Food Safety Engineering Research Center of Shaanxi Province, Xi'an, People's Republic of China
}

Submitted 25 February 2014: Final revision received 8 December 2014: Accepted 16 December 2014: First published online 16 March 2015

\begin{abstract}
Objective: To determine the breast-feeding pattern of four main ethnic groups (the Han, Uygur, Tibetan and Zhuang) living in rural western China.

Design: The study utilized a cross-sectional design.

Setting: Forty-five counties in ten provinces in western China in 2005.

Subjects: A sample of 11783 children younger than 36 months old (8960 Han, 1281

Uygur, 792 Tibetan and 750 Zhuang) and their mothers were recruited using a stratified, multistage, cluster random sampling method.

Results: The rates of exclusive breast-feeding of children at 6 months of age in the Han, Uygur, Tibetan and Zhuang ethnic groups were $11.6 \%, 0 \cdot 8 \%, 4.4 \%$ and $13.8 \%$, respectively. The rates of any breast-feeding for children at 24 months of age were $8.5 \%, 25.7 \%, 3.0 \%$ and $4.3 \%$ in the four ethnic groups, respectively. After adjusting for related factors, Zhuang children had a higher odds ratio of exclusive breast-feeding to 6 months compared with Han children, whereas Uygur and Tibetan children had lower odds ratio (Zhuang: OR $=1.291 ; 95 \%$ CI 1.006, 1.657; Uugur: OR=0.062; $95 \%$ CI 0.032, 0.121; Tibetan: OR =0.323; $95 \%$ CI 0.220, 0.475). Uygur children had a lower hazard ratio of discontinued breast-feeding compared with Han children, whereas Tibetan children had a higher hazard ratio (Uygur: HR=0.368; $95 \%$ CI 0.333, 0.408; Tibetan: HR=1.366; $95 \%$ CI 1.244 , 1.500).

Conclusions: The breast-feeding pattern differed among the Han, Uygur, Tibetan and Zhuang ethnic groups. The results suggest that health education regarding the benefits of breast-feeding is needed in rural western China.
\end{abstract}

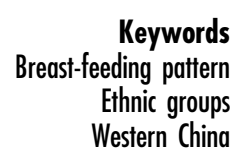

Breast milk is the most appropriate natural food for infants up to the age of 6 months, with breast-feeding offering health and developmental benefits to both babies and mothers $^{(1,2)}$. The WHO recommends exclusive breastfeeding for the first 6 months of life and continued breastfeeding up to 2 years of age or beyond ${ }^{(3)}$. Moreover, the Chinese government aims to have more than $50 \%$ of infants between 0 and 6 months old exclusively breast-feeding by $2020^{(4)}$. However, China has not achieved this goal and breast-feeding rates are still at relatively low levels. China is the most populous country in the world, with fifty-six ethnic groups. The largest ethnic group, the Han, accounts for more than $92 \%$ of China's population. The remaining fifty-five ethnic groups are collectively referred to as minorities and their socio-economic status is well below the average economic level in China. In China's western region, the minority populations are larger than in other regions and the ethnic groups in western China differ from one another with regard to their culture, social status, economic status, traditional living habits and customs.

Few studies have examined the breast-feeding practices of minorities in western China. This information is needed to determine the prevalence of breast-feeding in western China to make comparisons between the ethnic groups, investigate the possible reasons for different breastfeeding practices and provide evidence to support whether specific breast-feeding interventions are needed. The Chinese Ministry of Health and UNICEF completed a Rural Primary Health Care Project in rural western China from 2001 to 2005, which included forty-five counties in ten western provinces. The present study used programme data from 2005, with the objective to investigate the breastfeeding statuses of the Han, Uygur, Tibetan and Zhuang ethnic groups in rural western China.

\section{Methods}

\section{Participants and design}

The present cross-sectional study was part of the Rural Primary Health Care Project and was conducted in 2005 in rural western China, with a total of 14112 women and children from ten provinces enrolled. Informed consent was obtained from each adult participant at the start of 
the survey. The ethics committee at the Xi'an Jiaotong University College of Medicine approved the study. Considering the hierarchical structure of Chinese administrative districts and the imbalanced population distributions among the different provinces, a stratified, three-stage, probabilityproportional-to-size sampling method was employed for sampling in the present study. The counties were not selected randomly but were determined by the Chinese Ministry of Health and UNICEF. Five townships were randomly selected in each county; four villages were selected randomly in each township; and then a woman with children who were younger than 3 years old was selected from sixteen random families in each village. All of the women were interviewed in person by trained professional interviewers from the Xi'an Jiaotong University College of Medicine.

The sample numbers varied among the different ethnic groups in the present study. The Han, Uygur, Tibetan and Zhuang ethnic groups comprise the major ethnic groups in China, and their sample sizes were larger than those of other ethnic groups. Therefore we chose these four ethnic groups (Han, Uygur, Tibetan and Zhuang) to analyse differences in the breast-feeding practices of ethnic groups in western China.

\section{Control variables}

It was necessary to control for potential confounding factors to determine the effects of ethnicity on breast-feeding. Based on current knowledge and the nature of our data, we selected potential confounding factors from three groups of variables: socio-economics, children and mothers. The socio-economic factors included the household wealth index, which was constructed from an inventory of household assets, facilities or income resources using the principal component analysis method. This index was categorized into tertiles that indicated the poorest, middle class and wealthiest households. The children's factors included the child's sex and birth order. Finally, the mothers' factors included the education level, age at the child's birth, hospital delivery status and number of postpartum visits, as well as whether the mother cooked for the child alone and was given informational breast-feeding materials. We selected the control variables based on the following considerations: (i) whether these aforementioned factors affected the breastfeeding of the children (a factor that was primarily based on related studies ${ }^{(5-10)}$ ); and (ii) how these variables differed between the participants across ethnicities, which was determined by data analysis.

\section{Quality control}

The interviewers were trained before administering the survey. A pilot survey was performed before the formal survey to acquire the necessary information and to test the formal survey. During the survey, a checking system was instituted, including checking by oneself while in the field, checking by another interviewer and checking by the supervisor. The participants were re-interviewed when either logistical questions or missing values were found. The accurate age of each child was collected from the Permanent Residence Registration and/or the Record of Planned Immunization where the birth data were recorded.

\section{Feeding information and defining breast-feeding}

All of the participants were interviewed in person to collect the related data, including the children's health care, morbidity, anthropometry and feeding information as well as the mother's health care and other variables. Moreover, related information such as family background and the living environment (including altitudes) were also collected. When necessary, the fathers were asked to help the mothers provide family background information. A specially designed family questionnaire was used for the data collection. In the present study we primarily utilized the feeding data, which included breast-feeding practices (e.g. the time of initiation and the duration of breastfeeding) and complementary feeding practices (e.g. the time, frequency and types of introduced food). All feeding information was obtained by interviewing the mothers.

The following definitions of breast-feeding are used in the current paper ${ }^{(11,12)}$.

1. Any breast-feeding: the children received breast milk (either direct from the breast or expressed) with or without other drinks, formula or infant foods.

2. Exclusive breast-feeding: breast-feeding while giving no other foods or liquids (including water), with the exception of drops or syrups consisting of vitamins, mineral supplements or medicine. This type of breastfeeding was measured by the time when the mothers introduced other foods and fluids to their babies.

3. Predominant breast-feeding: in addition to breast milk, the infant may receive small amounts of culturally valued supplements, including water, water-based drinks, fruit juice and ritualistic fluids.

4. Full breast-feeding: includes both exclusive breastfeeding and predominant breast-feeding.

\section{Statistical analysis}

The sample sizes were 8960, 1281, 792 and 750 in the Han, Uygur, Tibetan, and Zhuang ethnic groups, respectively. Power was calculated to be $88 \%$ with a $95 \%$ confidence interval, assuming exclusive breast-feeding rates at the 6th month to be $11 \%$ and $15 \%$ in the Han and Zhuang children, respectively. A database was designed using EpiData version 3.02 and the data entry was duplicated. Frequency was used for the categorical variable descriptions. Either means and standard deviations or medians were used to describe the continuous variables. The $\chi^{2}$ test was performed for comparative analysis. The KaplanMeier model was employed to analyse the duration of breast-feeding and log-rank tests were used to test the 
Kaplan-Meier curves of the breast-feeding durations of children in the different ethnic groups. Non-conditional multivariate logistic regression was used to analyse ethnic differences in the exclusive breast-feeding rates for children younger than 6 months after adjusting for nine factors (i.e. the household wealth index, child gender, child birth order, mother's education level, maternal age at the child's birth, hospital delivery status, number of postpartum visits, whether the mother cooks for the child alone and whether the mother was given informational breast-feeding materials). After adjusting for these nine factors, Cox regression analysis was performed to analyse ethnic differences in breast-feeding duration. The level of significance was set at $P<0.05$. All analyses were performed with the SPSS statistical software package version $13 \cdot 0$.

\section{Results}

\section{Sociodemographic characteristics of participants}

The details of the sample and distribution of the major demographic variables are shown in Table 1. A total of 14112 women and children were recruited. Excluding children aged 3 years and older and other minorities with children, 11783 children (57.5\% male) and mothers were included in the present analysis. There were 8960, 1281, 792 and 750 Han, Uygur, Tibetan, and Zhuang children, respectively. The children were evenly distributed in the different age groups.

\section{Prevalence of breast-feeding}

The breast-feeding rates are detailed in Table 2 . The rate of exclusive breast-feeding at 6 months of age was low in the four ethnic groups reported here. It was highest among Zhuang infants at 6 months of age, but was only $13.8 \%$; and lowest among Uygur infants at 6 months of age, at $0 \cdot 8 \%$. The exclusive breast-feeding and full breast-feeding rates among infants declined by $50 \%$ from 4 to 6 months of age. The rate of any breast-feeding in all four ethnic groups at 12 months of age was greater than $50 \%$, with that in Uygur infants being above $90 \%$ at 12 months. At 24 months of age, less than $10 \%$ of the Han, Tibetan and Zhuang children were still fed breast milk, while $25.7 \%$ of the Uygur children were continuing to receive breast milk. Significant differences were found in the rates of exclusive and full breast-feeding at 4 months of age among the ethnic groups (both $P<0 \cdot 001$ ). No differences were found in exclusive breast-feeding rates and full breast-feeding rates at 6 months of age between the Han and Zhuang ethnic groups $(P=0.105$ and $P=0.567$, respectively), but significant differences were found in the rates of exclusive and full breast-feeding at 6 months of age among the other pairs of ethnic groups (comparison of exclusive breastfeeding rates and of full breast-feeding rates: Han $v$. Uygur, Han $v$. Tibetan, Uygur $v$. Tibetan, Uygur $v$. Zhuang and Tibetan $v$. Zhuang, $P<0 \cdot 001$ for all).

\section{Breast-feeding pattern in first 6 months}

We analysed the early breast-feeding patterns (i.e. during the first 6 months) of children older than 6 months in the four ethnic groups, as shown in Fig. 1. Mixed breastfeeding was the primary feeding pattern of Uygur children in their first 6 months of life. Among the Uygur children, $81.7 \%$ received complementary foods in the first month. Exclusive breast-feeding was the primary feeding pattern of Zhuang children in their first 6 months of life. Exclusive

Table 1 Sociodemographic characteristics of Han, Uygur, Tibetan and Zhuang ethnic groups in rural western China, 2005*

\begin{tabular}{|c|c|c|c|c|c|}
\hline Descriptor & Han $(n 8960)$ & Uygur ( $n$ 1281) & Tibetan ( $n$ 792) & Zhuang ( $n$ 750) & Total $(n 11783)$ \\
\hline Sample (\%) & $76 \cdot 0$ & $10 \cdot 9$ & $6 \cdot 7$ & $6 \cdot 7$ & $100 \cdot 0$ \\
\hline Male (\%) & $58 \cdot 7$ & $51 \cdot 8$ & $52 \cdot 1$ & $58 \cdot 1$ & 57.5 \\
\hline \multicolumn{6}{|l|}{ Age group (\%) } \\
\hline $0-<6$ months & $13 \cdot 2$ & $17 \cdot 3$ & $10 \cdot 1$ & $18 \cdot 7$ & 13.8 \\
\hline $6-<12$ months & $22 \cdot 7$ & $20 \cdot 6$ & $20 \cdot 6$ & $26 \cdot 7$ & $22 \cdot 6$ \\
\hline $12-<24$ months & $36 \cdot 1$ & 35.8 & 38.5 & 38.9 & $36 \cdot 4$ \\
\hline $24-<36$ months & $28 \cdot 0$ & $26 \cdot 3$ & $30 \cdot 8$ & $15 \cdot 7$ & $27 \cdot 2$ \\
\hline \multicolumn{6}{|l|}{ Child's birth order (\%) } \\
\hline 1 & $61 \cdot 7$ & $48 \cdot 0$ & $58 \cdot 0$ & $68 \cdot 2$ & $60 \cdot 3$ \\
\hline$\geq 2$ & $38 \cdot 3$ & $52 \cdot 0$ & $42 \cdot 0$ & $31 \cdot 8$ & 39.7 \\
\hline \multicolumn{6}{|l|}{ Maternal education (\%) } \\
\hline 0 years & $7 \cdot 1$ & 4.0 & $29 \cdot 8$ & 4.0 & 8.0 \\
\hline $1-9$ years & $84 \cdot 0$ & 93.3 & $64 \cdot 7$ & $90 \cdot 4$ & $84 \cdot 1$ \\
\hline$>9$ years & $9 \cdot 0$ & $2 \cdot 7$ & 5.5 & $5 \cdot 6$ & 7.8 \\
\hline \multicolumn{6}{|l|}{ Maternal age at child's birth (\%) } \\
\hline$<20$ years & 4.3 & 21.5 & $12 \cdot 1$ & 3.5 & $6 \cdot 7$ \\
\hline $20-29$ years & $75 \cdot 7$ & $66 \cdot 0$ & 74.7 & $74 \cdot 3$ & 74.4 \\
\hline$\geq 30$ years & $20 \cdot 0$ & $12 \cdot 6$ & 13.2 & $22 \cdot 2$ & 18.9 \\
\hline \multicolumn{6}{|l|}{ Main source of household income (\%) } \\
\hline Farming or animal husbandry only & $42 \cdot 2$ & $76 \cdot 0$ & $52 \cdot 8$ & $54 \cdot 5$ & $47 \cdot 4$ \\
\hline Farming or animal husbandry and others & 57.8 & $24 \cdot 0$ & $47 \cdot 2$ & $45 \cdot 5$ & $52 \cdot 6$ \\
\hline
\end{tabular}

*In comparison of baseline characteristics of children enrolled in the study, there were significant differences across all of the characteristics among the four ethnic groups $(P<0.05)$. 
Table 2 Breast-feeding rates among Han Ethnic, Uygur, Tibetan and Zhuang children in rural western China, 2005

\begin{tabular}{|c|c|c|c|c|c|}
\hline Breast-feeding rate & Month & Han & Uygur & Tibetan & Zhuang \\
\hline \multirow[t]{2}{*}{ Exclusive breast-feeding (\%)* } & 4 & 29.8 & 1.9 & $8 \cdot 6$ & 60.5 \\
\hline & 6 & 11.6 & 0.8 & 4.4 & 13.8 \\
\hline \multirow[t]{2}{*}{ Full breast-feeding (\%)† } & 4 & $55 \cdot 4$ & $10 \cdot 1$ & $33 \cdot 8$ & $73 \cdot 1$ \\
\hline & 6 & $27 \cdot 3$ & $5 \cdot 1$ & $16 \cdot 9$ & $26 \cdot 3$ \\
\hline \multirow{4}{*}{ Any breast-feeding (\%)‡ } & 4 & $95 \cdot 2$ & $97 \cdot 6$ & 95.9 & 97.4 \\
\hline & 6 & $92 \cdot 8$ & $97 \cdot 0$ & $92 \cdot 6$ & 97.0 \\
\hline & 12 & $62 \cdot 2$ & $93 \cdot 6$ & $50 \cdot 2$ & 63.6 \\
\hline & 24 & 8.5 & $25 \cdot 7$ & 3.0 & $4 \cdot 3$ \\
\hline
\end{tabular}

*Exclusive breast-feeding: breast-feeding while giving no other foods or liquids (including water), with the exception of drops or syrups consisting of vitamins, mineral supplements or medicine. Exclusive breast-feeding rate at $X$ months = number of children aged $\geq X$ months who are exclusively breast-feeding to $X$ months/total number of children aged $\geq X$ months.

†Full breast-feeding: includes exclusive breast-feeding and predominant breast-feeding (predominant breast-feeding: in addition to breast milk, the infant may receive small amounts of culturally valued supplements, including water, water-based drinks, fruit juice and ritualistic fluids). Full breast-feeding rate at $X$ months $=$ number of children aged $\geq X$ months who are fully breast-feeding to $X$ months/total number of children aged $\geq X$ months.

$\ddagger$ Any breast-feeding: the child received breast milk (direct from the breast or expressed) with or without other drinks, formula or infant foods. Any breast-feeding rate at $X$ months $=$ number of children aged $\geq X$ months receiving any breast-feeding to $X$ months/total number of children aged $\geq X$ months.
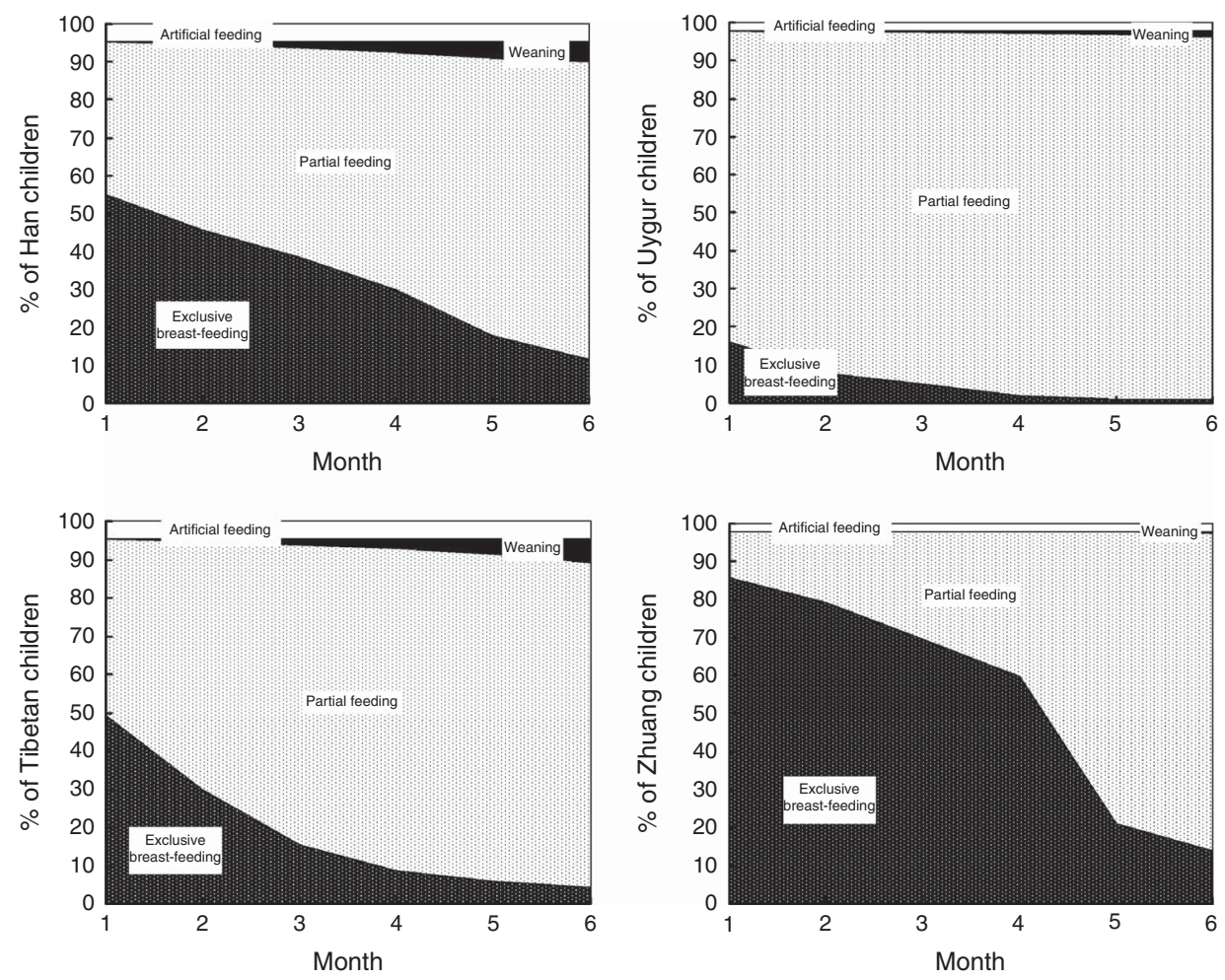

Fig. 1 Comparison of feeding patterns among Han, Uygur, Tibetan and Zhuang children in rural western China, 2005

breast-feeding was also the primary feeding practice of the Han and Tibetan children in the first month, but the practice shifted to mixed breast-feeding after the first month. Differences were found in the rates of exclusive breast-feeding among the ethnic groups for children in the first 1, 2, 3, 4, 5 and 6 months of life (all $P<0 \cdot 001$ ).

\section{Duration of breast-feeding}

The three main weaning ages were at 12, 18 and 24 months. The Uygur children tended to be weaned later than the Han, Tibetan and Zhuang children (Fig. 2). Most of the children in the Han, Tibetan and Zhuang ethnic groups were breast-fed for 12 months, but the Uygur children were breast-fed for significantly longer compared with the other three ethnic groups $(P<0 \cdot 001)$. The median planned weaning age was 12 months for the Han, Tibetan and Zhuang children, and the median planned weaning age was 24 months for the Uygur children. The median actual weaning age in months followed this trend within the four ethnic groups (Table 3).

\section{Results of multivariate logistic regression and multivariate Cox regression}

The factors that were most likely to be associated with breast-feeding were entered in the multivariate logistic 


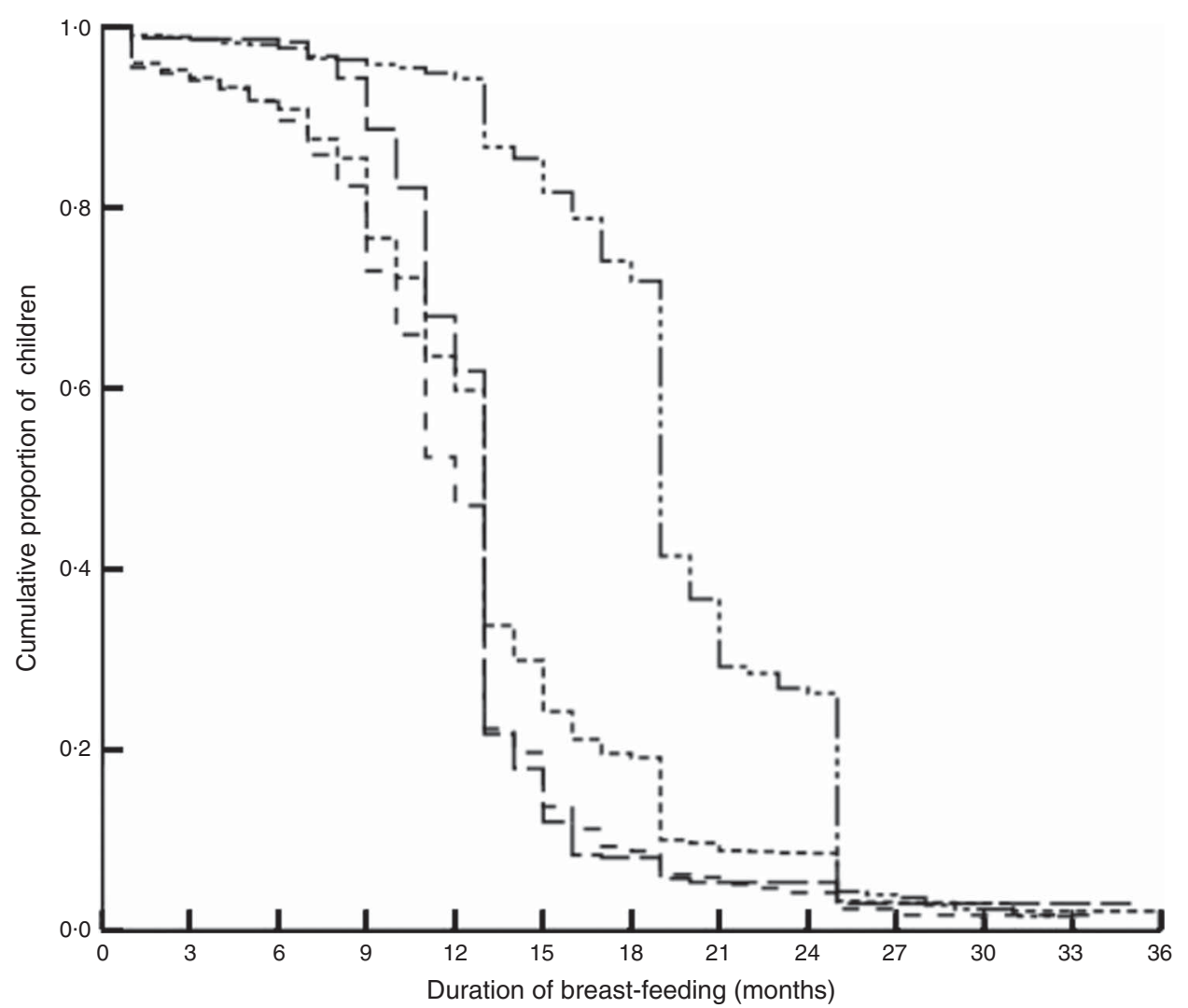

Fig. 2 Comparison of duration of breast-feeding among Han (- - - -), Uygur ( - - - - ), Tibetan (- - - -) and Zhuang (- - - ) children in rural western China, 2005

Table 3 Planned weaning age and actual weaning age among Han, Uygur, Tibetan and Zhuang children in rural western China, 2005

\begin{tabular}{lccccr}
\hline & Han & Uygur & Tibetan & Zhuang & $P$ value \\
\hline Planned weaning age (months), median & 12 & 24 & 12 & 12 & $<0.001$ \\
Actual weaning age (months), mediant & 12 & 18 & 10 & 12 & $<0.001$ \\
\hline
\end{tabular}

${ }^{*}$ Planned weaning age was obtained by interview from mothers who continued breast-feeding.

†Actual weaning age was obtained by interview from weaned children's mothers.

Table 4 Predictor of exclusive breast-feeding to 6 months among children under 36 months old in rural western China, 2005: result from logistic regression*

\begin{tabular}{lllllr}
\hline & & & \multicolumn{2}{c}{$95 \% \mathrm{Cl}$ for OR } & \\
\cline { 5 - 6 } & & OR & Lower & Upper & $P$ value \\
\hline Ethnic group & Han & 1 & - & - & - \\
& Uygur & 0.062 & 0.032 & 0.121 & $<0.001$ \\
& Tibetan & 0.323 & 0.220 & 0.475 & $<0.001$ \\
& Zhuang & 1.291 & 1.006 & 1.657 & 0.045 \\
\hline
\end{tabular}

*Nine factors were adjusted for in logistic regression (household wealth index, child's gender, child's birth order, mother's education level, maternal age at the child's birth, hospital delivery status, number of postpartum visits, cooking for the child alone and whether the mother was given informational breast-feeding materials).

regression model. After adjusting for the nine factors (i.e. household wealth index, child's gender, child's birth order, mother's education level, maternal age at the child's birth, hospital delivery status, number of postpartum visits, cooking for the child alone and whether the mother was given informational breast-feeding materials), Zhuang children had a significantly higher odds ratio of exclusive breast-feeding to 6 months compared with Han children (OR $=1.291 ; 95 \%$ CI 1.006, 1.657, $P=0.045$ ), whereas Uygur children and Tibetan children had a significantly lower odds ratio of exclusive breast-feeding to 6 months compared with Han children (Uygur: OR $=0.062 ; 95 \%$ CI 0.032, 0.121, $P<0 \cdot 001$; Tibetan: OR $=0 \cdot 323 ; 95 \%$ CI 0.220, $0 \cdot 475, P<0 \cdot 001$; Table 4). 
Table 5 Predictor of discontinuing any breast-feeding among children under 36 months old in rural western China, 2005: result from Cox regression*

\begin{tabular}{lllllr}
\hline & & & \multicolumn{2}{c}{$95 \% \mathrm{Cl}$ for HR } & \\
\cline { 5 - 6 } & & HR & Lower & Upper & $P$ value \\
\hline Ethnic group & Han & 1 & - & - & - \\
& Uygur & 0.368 & 0.333 & 0.408 & $<0.001$ \\
& Tibetan & 1.366 & 1.244 & 1.500 & $<0.001$ \\
& Zhuang & 1.100 & 0.990 & 1.221 & 0.075 \\
\hline
\end{tabular}

HR, hazard ratio.

*Nine factors were adjusted for in logistic regression (household wealth index, child's gender, child's birth order, mother's education level, maternal age at the child's birth, hospital delivery status, number of postpartum visits, cooking for the child alone and whether the mother was given informational breast-feeding materials).

The factors likely to be associated with breast-feeding were entered into the multivariate Cox regression model. After adjusting for these factors, Uygur children had a significantly lower hazard ratio of discontinued breastfeeding compared with Han children ( $\mathrm{HR}=0 \cdot 368,95 \% \mathrm{CI}$ 0.333, 0.408, $P<0.001$ ), whereas the Tibetan children had a significantly higher hazard ratio of discontinued breastfeeding compared with Han children ( $\mathrm{HR}=1 \cdot 366,95 \% \mathrm{CI}$ 1.244, 1.500, $P<0 \cdot 001$; Table 5).

\section{Discussion}

The present study compares the breast-feeding practices of four ethnic groups in rural western China in 2005 and describes the differences among these four groups. The results revealed that the breast-feeding rates and durations were different among the four ethnic groups.

The study focused on the breast-feeding rates of four ethnic groups. The Zhuang children had the highest exclusive breast-feeding rate and full breast-feeding rate among children at ages 4 and 6 months, whereas the Uygur children had the lowest exclusive breast-feeding rate and full breast-feeding rate at the same ages. All of the ethnicities showed a sharp decline from 4 to 6 months in the rates of exclusive and full breast-feeding. The exclusive breast-feeding and full breastfeeding rates of the Han children were at the median level compared with the other ethnicities. The exclusive and full breast-feeding rates of the Tibetan children were lower than those of the Zhuang and Han children. The study results are consistent with a study in Xinjiang in 2006. In that longitudinal study, among the Uygur, Han and other minority groups (including the Kazakh, Xibe and Hui), the Uygur children were least likely to be exclusively breast-fed and the Han children were the median group in terms of exclusive breastfeeding and full breast-feeding rates for infants 6 months of age $^{(13)}$. However, the Uygur children had a longer duration of breast-feeding than the other ethnic groups. The rates of any breast-feeding among the Uygur children at ages 12 and 24 months were higher than those of the other ethnic groups, and the weaning age of the weaned Uygur children was much older compared with the other ethnic groups.
The artificial feeding and weaning rates of the four ethnic groups were all low in the first 6 months. The mixed feeding rate of the Uygur children was greater than that of the other ethnicities in the first 6 months. The Uygur mothers were more likely to give early complementary foods to their children and more than $80 \%$ of the Uygur children were fed complementary foods in the first month. Early complementary feeding is the main reason for the low exclusive breast-feeding rate of the Uygur children. In the present study, we found that 'the age should be weaned is coming' was the primary reason to discontinue breast-feeding. Uygur mothers' planned age to wean their children was much older than that of mothers from other ethnic groups. Accordingly, the Uygur children had a longer duration of breast-feeding than the children of the other ethnicities. Mixed feeding was the primary feeding pattern of the Tibetan children in the first 6 months and these children also had a short duration of breast-feeding. Most of the Tibetan children had been fed zanba (a traditional Tibetan food that is a semi-solid or solid mixture of cooked highland barley power and butter tea) early. For example, Tibetan children were introduced to zanba at approximately 1 month of age; by 4 months, more than $80 \%$ of the Tibetan children had been fed $z a n b a^{(14)}$. The Han children were characterized by a lower rate of exclusive breast-feeding and a shorter duration of breastfeeding. Han mothers often offered water and sugar water to babies as a universal complementary food in the first 6 months of life and thought that the baby should be weaned before 12 months. The exclusive breast-feeding rate for the Zhuang children in the first 6 months was the highest among the four ethnic groups, but the Zhuang children had a shorter duration of breast-feeding. Zhuang mothers planned to wean their babies at 12 months of age, which is younger than the planned weaning age of Uygur mothers.

The result of the multivariate logistical regression revealed that Zhuang children were still more likely to be exclusively breast-fed at 6 months compared with the other children, and Uygur children and Tibetan children were less likely to be exclusively breast-fed at 6 months compared with Han children (after adjusting for the nine related factors). The same adjustments revealed that the Uygur children had a lower hazard ratio of discontinued breast-feeding, and the 
Tibetan children had a higher hazard ratio of discontinued breast-feeding, compared with Han children in the multivariate Cox regression analysis. The socio-economic factors that influence breast-feeding practices have been extensively described in the literature and widely reviewed ${ }^{(15-19)}$, but the breast-feeding differences between ethnic groups could not be exclusively explained by socio-economic characteristics such as education and economic status in the present study; the breast-feeding differences among the ethnicities were more likely related to different traditional feeding habits within the ethnic groups. Additional studies that focus on different feeding cultures among the ethnic groups are needed to provide a deeper understanding of the reasons for different feeding patterns.

The main issue of breast-feeding among the different ethnic groups in western China is the low prevalence of exclusive breast-feeding and full breast-feeding as well as the short duration of breast-feeding, all of which are below the Chinese and international targets ${ }^{(4,20)}$. We recommend that Uygur mothers are provided feeding education about the benefits of exclusive breast-feeding for the first 6 months. Tibetan, Zhuang and Han mothers would benefit from a feeding education to develop a longer duration of breast-feeding.

The data analysed in the present study were collected from the most undeveloped rural areas in western China. The strength of the current study is its large number of participants. However, there are several limitations that must be considered when interpreting the study results. First, there was recall bias because all feeding information was obtained through mothers' recall. Especially, there was also potential for greater recall bias on breast-feeding the more time that has passed between cessation and recall. Second, there may have been other unobserved confounding factors, such as occupation of the mothers, which could not be controlled in multivariate analysis because they were not available in this survey. Third, this was a cross-sectional not a prospective study and the infants were at different ages at the time of the survey, which might have affected accurate estimation of the magnitude of breast-feeding.

\section{Conclusion}

The exclusive breast-feeding rates for children younger than 6 months and the continued breast-feeding rates for children at 2 years of age differed among the Han, Uygur, Tibetan and Zhuang ethnic groups, and were far below the WHO recommendations. Additionally, mothers in western China require feeding education about the benefits of exclusive breast-feeding for 6 months and a longer duration of breast-feeding.

\section{Acknowledgements}

Acknowledgements: The authors thank the Ministry of Health (MOH), People's Republic of China and UNICEF for their support and cooperation; the Health Development Department of each province in the project, the local health bureaus and the maternal and child health stations, for their cooperation and organization in collecting the field data; and the staff from Xi'an Jiaotong University for their assistance with the data collection. Financial support: Financial support was provided by the $\mathrm{MOH}$ and UNICEF (grant number YH001), and the National Natural Science Foundation of China (grant number 81230016). $\mathrm{MOH}$ and UNICEF selected the counties to be sampled in the present study, but had no role in the design, analysis or writing of this article. Conflict of interest: None. Authorship: P.Q. designed the study, collected the data, conducted the data analysis and prepared the manuscript. P.Q had full access to all data in the study and assumed responsibility for the integrity of the data and the accuracy of the data analysis. T.W. designed the study and the analysis and prepared the manuscript. F.L. designed the study and the analysis and prepared the manuscript. S.D. assisted with the data analysis and reviewed the manuscript. L.Z. assisted with the data analysis and reviewed the manuscript. H.Y. designed the study and the analysis and prepared the manuscript. Ethics of human subject participation: The ethics committee at the Xi'an Jiaotong University College of Medicine approved the study.

\section{References}

1. WHO Collaborative Study Team on the Role of Breastfeeding on the Prevention of Infant Mortality (2000) Effect of breastfeeding on infant and child mortality due to infectious diseases in less developed countries: a pooled analysis. Lancet 355, 451-455.

2. Binns CW, Lee M \& Scott JA (2001) The fetal origins of disease hypothesis: public health implications for the AsiaPacific region. Asia Pac J Public Health 13, 68-73.

3. World Health Organization (2001) The optimal duration of exclusive breastfeeding. Report of the expert consultation. http://www.who.int/nutrition/publications/infantfeeding/ WHO_NHD_01.09/en/ (accessed March 2001).

4. The Central People's Government of the People's Republic of China (2011) National Program of Action for Child Development in China (2010-2020). http://www.gov. cn/zwgk/2011-08/08/content_1920457.htm (accessed July 2011).

5. Lee HJ, Rubio MR, Elo IT et al. (2005) Factors associated with intention to breastfeed among low-income, inner-city pregnant women. Matern Child Hlth J 9, 253-261.

6. Thulier D \& Mercer J (2009) Variables associated with breastfeeding duration. J Obstet Gynecol Neonatal Nurs 38, 259-268.

7. Glassman ME, McKearney K, Saslaw M et al. (2014) Impact of breastfeeding self-efficacy and sociocultural factors on early breastfeeding in an urban, predominantly dominican community. Breastfeed Med 9, 301-307.

8. Gianni ML, Roggero P, Amato O et al. (2014) Intervention for promoting breast milk use in neonatal intensive care unit: a pilot study. J Matern Fetal Neonatal Med 27, 475-478.

9. Esteves TMB, Daumas RP, de Oliveira MIC et al. (2014) Factors associated to breastfeeding in the first hour of life: systematic review. Rev Saude Publica 48, 697-708. 
10. Castro PD, Layte R \& Kearney J (2014) Ethnic variation in breastfeeding and complimentary feeding in the Republic of Ireland. Nutrients 6, 1832-1849.

11. Labbok M \& Krasovec K (1990) Toward consistency in breastfeeding definitions. Stud Fam Plann 21, 226-230.

12. Colin WB \& Scott JA (2002) Breastfeeding: reasons for starting, reasons for stopping and problems along the way. Breastfeed Rev 10, 13-19.

13. Xu FL, Binns C, Nazi G et al. (2006) A comparison of breastfeeding among Han, Uygur and other ethnic groups in Xinjiang, PR China. BMC Public Health 6, 196.

14. Dang S, Yan H, Yamamoto S et al. (2005) Feeding practice among younger Tibetan children living at high altitudes. Eur J Clin Nutr 59, 1022-1029.

15. Simard I, O'Brien HT, Beaudoin A et al. (2005) Factors influencing the initiation and duration of breastfeeding among low-income women followed by the Canada Prenatal Nutrition Program in 4 regions of Quebec. J Hum Lact 21, 327-337.
16. Dennis CL (2002) Breastfeeding initiation and duration: a 1990-2000 literature review. J Obstet Gynecol Neonatal Nurs 31, 12-32.

17. Ceti AC, Rich-Edwards JW, Richardson MK et al. (2005) Immigration, race/ethnicity, and social and economic factors as predictors of breastfeeding initiation. Arch Pediatr Adolesc Med 159, 255-260.

18. Noughabi ZS, Tehrani SG, Foroushani AR et al. (2014) Prevalence and factors associated with exclusive breastfeeding at 6 months of life in Tehran: a populationbased study. East Mediterr Health J 20, 24-32.

19. Maastrup R, Hansen BM, Kronborg H et al. (2014) Breastfeeding progression in preterm infants is influenced by factors in infants, mothers and clinical practice: the results of a national cohort study with high breastfeeding initiation rates. PLoS One $\mathbf{9}$, e108208.

20. Habicht JP (2004) Expert consultation on the optimal duration of exclusive breastfeeding: the process, recommendations, and challenges for the future. Adv Exp Med Biol 554, 79-87. 